\title{
Influence of number of drugs on olfaction in the elderly*
}

\author{
Giancarlo Ottaviano', Enrico Savietto', Bruno Scarpa², Anna Bertocco3, \\ Pietro Maculan', Giuseppe Sergi³, Alessandro Martini', Enzo Manzato3, \\ Gino Marioni
}

Rhinology 56: 4, 351-357, 2018

https://doi.org/10.4193/Rhin17.152

*Received for publication:

July 12, 2017

Accepted: January 26, 2018

Department of Statistical Sciences, University of Padova, Padova, Italy

${ }^{3}$ Geriatric Unit, Department of Medicine, University of Padova, Padova, Italy

Background: The etiology of age-related olfactory loss is still unclear, but it has been claimed that polypharmacotherapy may contribute to olfactory dysfunction, particularly in the elderly, who are more likely to need multiple drugs. The present pilot study investigated the relationship between smell and the number and type of drugs taken in a group of elderly.

Methodology: 50 elderly volunteers ( $\geq 65$ years old) who were healthy from the sinonasal standpoint (SNOT $22<1$ ) and had no cognitive impairments [Mini Mental State Examination (MMSE) > 18] were administered the Screening 12 test $^{\oplus}$ and tested on their $\mathrm{n}$-butanol olfactory threshold. Their olfactory performance was then connected with the number and type of drugs participants used.

Results: The mean age of the included volunteers was $74 \pm 7$ years. No association emerged between odor identification and number of drugs taken. The number of drugs taken correlated directly with a worse olfactory threshold and with a worse MMSE score, meaning a worse cognitive status. Odor identification significantly worsened with age. Comparing those volunteers taking only one drug known to not influence olfaction with another sub-group of volunteers taking five or more drugs, it was evident that subjects taking only one drug scored significantly better in olfactory threshold test and MMSE, and marginally better in olfactory identification test. For what concerns the difference between male and female volunteers, no difference in olfactory test result was shown, both for threshold and identification. Univariate analysis showed a direct correlation between the consumption of calcium channel blockers, ß-blockers, acetylsalicylic acid and olfactory threshold, meaning a worse sense of smell. Acetylsalicylic acid also correlated inversely with odor identification, meaning again a worse sense of smell, and so did potassium-sparing diuretics. Multivariate analysis showed that MMSE scores correlated with a better sense of smell, that is a lower olfactory threshold, and that a-blockers and acetylsalicylic acid negatively affected olfactory threshold, meaning a worse sense of smell. Acetylsalicylic acid also correlated inversely with odor identification, meaning again a worse sense of smell.

Conclusions: The number of drugs taken demonstrated to be significantly correlated with a worse olfactory threshold and worse MMSE. Larger studies on elderly volunteers are needed to confirm these preliminary findings.

Key words: smell, drugs, elderly, olfactory threshold, olfactory identification, Screening 12 test

\section{Introduction}

Olfaction is very important for orientation, feeding and hazard recognition. Sadly, our sense of smell decreases with age: half of the US population between the ages of 65 and 80 years, and about $75 \%$ of those over 80 years old experience olfactory loss $^{(1)}$. A recent European study, based on a cross-sectional survey, analyzing the learning process during life of the sense of smell, showed that olfaction in women is better than in men through all ages ${ }^{(2)}$. Olfactory loss in elderly will be an increasingly severe problem as the global population grows older, with the numbers of people over 65 expected to double in the US, for instance, by 2030 (based on US Census estimates) ${ }^{(3-6)}$. The etiology of age-related olfactory loss is largely unknown $n^{(7)}$. Aging itself naturally coincides with changes in the olfactory 
epithelium, more limited mucus secretions, and loss of olfactory neurons secondary to a diminished capacity for their regeneration $^{(8)}$. An association between age, neurodegenerative disorders, and olfactory dysfunction has recently been reported ${ }^{(8)}$. Another potential reason for olfactory dysfunction may relate to people's general health and the number and types of drugs they take ${ }^{(9)}$. As the elderly are the most exposed to multiple drug consumption (given their higher morbidity rate) $)^{(10)}$, polypharmacotherapy may reasonably contribute to olfactory dysfunction in this age group. Negative effects of drugs on chemosensation have already been described, but most investigations on this topic focused on taste, while there remains a dearth of information regarding the sense of smell ${ }^{(9,11)}$.

Since no definitive conclusions can be drawn as yet on the role of drugs "possibly" or "probably" influencing olfaction in altering the sense of smell in humans, the present study was designed to test the olfactory threshold and odor identification of a cohort of elderly volunteers in order to elucidate the relationship between their olfaction and the number and types of drugs they were taking.

\section{Materials and methods}

\section{Study population}

The present investigation was conducted in accordance with the 1996 Helsinki Declaration and was approved in 2016 by our Sections in-house committee. Written informed consent was obtained from each volunteer before starting any study-related procedure. All volunteers enrolled in the study were asked to complete the SNOT 22 questionnaire ${ }^{(12)}$ and the Mini Mental State Examination (MMSE) (with scores ranging from 0 to 30$)^{(10)}$. The crude MMSE scores were adjusted for age and formal education using the coefficients proposed for the Italian population ${ }^{(13)}$. All volunteers enrolled were $\geq 65$ years old.

\section{Study design}

The main exclusion criteria adopted for the present investigation were: previous sino-nasal surgery, allergic or non-allergic

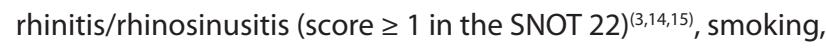
a history of previous post-viral loss of smell, head trauma, cognitive impairment (MMSE score $<18)^{(10)}$ a diagnosis of diabetes or hypothyroidism ${ }^{(16)}$. Heavy drinkers (defined as daily consumers of 5 or more standard alcoholic beverages containing 14 grams of pure alcohol each for men, and 4 or more for women, according to the definition of the National Institute on Alcohol Abuse and Alcoholism), individuals on chemotherapy, or diagnosed with cancer or previously treated with head and neck radiotherapy were also ruled out. Other exclusion criteria were long-term treatments with aminoglycosides or tetracycline, and any use of opioids, cannabinoids or sildenafil, which are known to affect olfaction ${ }^{(9)}$.

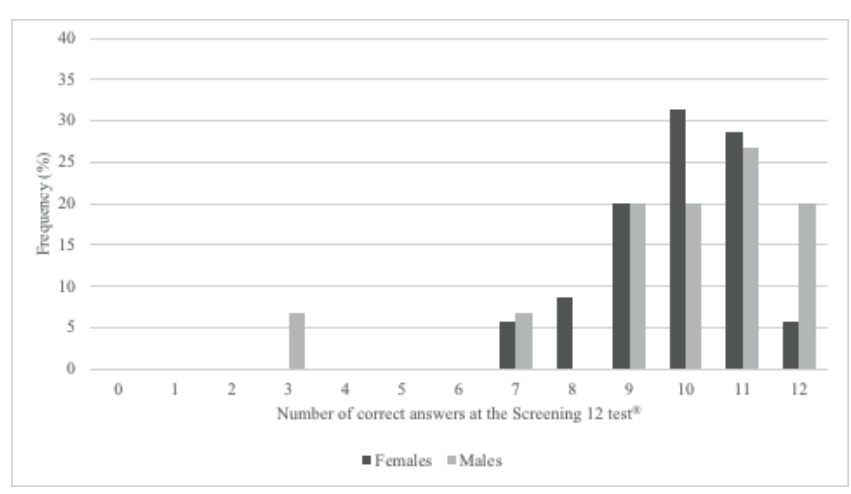

Figure 1. The frequency of the correct answers at the Screening 12 test $^{\oplus}$, separately for males and females.

\section{Outcomes}

Screening 12 test ${ }^{\oplus 17,18)}$ and Sniffin' Sticks n-butanol olfactory threshold subtest ${ }^{(19,20)}$ were performed from each volunteer. Age, sex, height, weight, body mass index (BMI), and both the number and the types of drugs being taken were also recorded. Olfactory performance was then correlated with the number and type of drugs participants used.

\section{Statistical analysis}

Linear regression models were used to examine the influence of the number of drugs taken on a participant's olfactory threshold and odor identification, taking the effect of other variables available into account. A simple regression model was used to assess the univariate effect of the number of drugs on both the olfactory threshold and odor identification. A multiple regression model was also developed, including all the variables considered (number of drugs, sex, age, height, BMI, and MMSE score). The variables for the model were selected by means of a regression on all subsets ${ }^{(21)}$, a method that identifies the best model in accordance with the Akaike information criterion (AIC). The goodness of fit and the validity of the model were ascertained by means of a graphical analysis of the residuals and the computation of appropriate indices $\left(\chi^{2}\right.$, test $\left.F\right)$.

A Welch test was used to evaluate the differences in olfactory outcomes between males and females. The same test was used to analyze the olfactory outcome differences between a subgroup taking only one drug known to not influence olfaction and another sub-group of volunteers taking more than 5 drugs. A p-value $<0.05$ was considered statistically significant. All analyses were run using the $\mathrm{R}$ language and environment for statistical computing (R Foundation for Statistical Computing, Vienna, Austria).

\section{Results}

A cohort of 50 elderly volunteers (35 females, 15 males; mean age $74 \pm 7$ years) was recruited at Padova University Hospital (see Table 1 for detailed clinical and demographic characteristics). 
Table 1. Clinical, demographic variables of the cohort of volunteers, separated for males and females.

\begin{tabular}{|c|c|c|c|c|c|c|c|c|c|}
\hline \multirow[b]{2}{*}{ Variable } & \multirow[b]{2}{*}{ Mean } & \multicolumn{2}{|c|}{ Males $(n=15)$} & \multicolumn{6}{|c|}{ Females $(n=35)$} \\
\hline & & SD & Range & Normal value & Mean & SD & Range & Normal value & p-value* \\
\hline Age $(y r)$ & 73 & 6.9 & $66-86$ & - & 75 & 7.5 & $65-91$ & - & 0.2911 \\
\hline Height $(\mathrm{cm})$ & 175 & 8.1 & 163-186 & - & 160 & 5.2 & $147-170$ & - & $<0.0001$ \\
\hline Weight (kg) & 79 & 9.6 & $60-96$ & - & 63 & 13.6 & 41.6-106 & - & $<0.0001$ \\
\hline BMI & 26 & 2.8 & $22.3-30.5$ & - & 25 & 5.4 & $17.6-44.1$ & - & 0.3135 \\
\hline Number of drugs taken & 4 & 1.4 & $1-11$ & - & 4 & 2.5 & $1-11$ & - & 0.5821 \\
\hline Olfactory identification & 10 & 1.4 & $7-12$ & $11.33 \pm 0.48^{(37)}$ & 10 & 1.3 & $7-12$ & $11.44 \pm 0.53^{(37)}$ & 0.9298 \\
\hline Olfactory threshold & 4 & 1.7 & $1.5-6-5$ & $7.15 \pm 3.59^{(38)}$ & 5 & 1.5 & $1.5-7.5$ & $7.44 \pm 3.51^{(38)}$ & 0.1016 \\
\hline MMSE & 29 & 0.9 & $28-30$ & $27.0 \pm 2.4^{(13)}$ & 28 & 2.5 & $21-30$ & $27.0 \pm 2.4^{(13)}$ & 0.0101 \\
\hline
\end{tabular}

Adjusted Mini Mental State Examination (MMSE). Body mass index (BMI). * Welch test

Table 2. Univariate analysis: correlations between olfactory threshold and odor identification with the demographic/clinical variables considered.

\begin{tabular}{ccccccccc} 
Parameters & No. of drugs & MMSE & Weight $(\mathbf{k g})$ & Height $(\mathbf{c m})$ & BMI & Age (yrs) & Sex \\
Olfactory threshold & $r^{2}=0.22$ & $r^{2}=0.02$ & $r^{2}=0.06$ & $r^{2}=0.03$ & $r^{2}=0.015$ & $r^{2}=0.06$ & $r^{2}=0.06$ \\
& $p=0.0003$ & $p=0.15$ & $p=0.08$ & $p=0.13$ & $p=0.38$ & $p=0.09$ & $p=0.08$ \\
\multirow{2}{*}{ Odor identification } & $r^{2}=0.008$ & $r^{2}=0.05$ & $r^{2}=0.02$ & $r^{2}=0.02$ & $r^{2}=0.02$ & $r^{2}=0.12$ & $r^{2}=0.0003$ & $p=0.9$
\end{tabular}

Adjusted Mini Mental State Examination (MMSE). Body mass index (BMI).

Table 3. Multivariate regression model: correlations between olfactory threshold and the demographic/pharmacological variables considered.

\begin{tabular}{|lcccc|}
\hline \multicolumn{1}{c}{ Coefficients } & & & & \\
& Estimate & Std. Error & t value & p-value \\
\hline (Intercept) & 0.84791 & 2.96516 & 0.286 & 0.776 \\
\hline BMI & -0.08354 & 0.04264 & -1.959 & 0.057 \\
\hline MMSE & 0.22261 & 0.10466 & 2.127 & 0.039 \\
\hline a-blockers & -1.80306 & 0.84006 & -2.146 & 0.038 \\
\hline Sartans & 0.81235 & 0.48588 & 1.672 & 0.102 \\
\hline Dicumarolics & -1.57107 & 0.78277 & -2.007 & 0.051 \\
\hline Antiplatelet & -1.30764 & 0.44553 & -2.935 & 0.005 \\
drugs & 1.42327 & 0.74372 & 1.914 & 0.063 \\
\hline Biguanides & & & & \\
\hline
\end{tabular}

Adjusted Mini Mental State Examination (MMSE). Body mass index (BMI).

Figure 1 reports the frequency of the correct answers of the Screening 12 test $^{\oplus}$.

Although no association was found between odor identification and the number of drugs being taken $(p=0.53)$, there was a correlation between the number of drugs taken and both a worse olfactory threshold $\left(r^{2}=0.22, p=0.0003\right)$ and a worse MMSE score $\left(r^{2}=0.11, p=0.012\right)$. No correlation emerged between olfactory threshold and MMSE score $\left(r^{2}=0.02, p=0.15\right)$ (Table 2). Statistical analysis revealed an inverse correlation between age and odor identification $\left(r^{2}=0.12, p=0.01\right)$, but ruled out any correlation between the other variables considered (sex, weight, height, $\mathrm{BMI}$ ) and either olfactory threshold or odor identification (Table 2).

To study the effect of the drugs on olfaction in the elderly, ideally we would have preferred to have a control group composed from volunteers not taking any medication. Anyway, this condition is very difficult to obtain in elderly, so we built a control sub-group of ten volunteers taking only 1 medication known to have no influence on olfaction and a study sub-group of volunteers taking more than 5 medications. The group taking $>5$ drugs showed a significantly worse olfactory threshold $(p=0.00047)$ and a marginally significant worse olfactory identification score $(p=0.08$ ) (Figure $2 a$ and $2 b$, respectively). Furthermore, the group taking $>5$ drugs showed a significantly worse MMSE score ( $p=0.007$ ) (Figure $2 c)$.

Analyzing the relationship between the types of medication being taken and participants' sense of smell, univariate analysis showed a correlation between a worse olfactory threshold and the consumption of calcium channel blockers $\left(r^{2}=0.10, p=0.02\right)$, B-blockers ( $\left.r^{2}=0.09, p=0.03\right)$, and antiplatelet drugs, namely acetylsalicylic acid $\left(r^{2}=0.18, p=0.0012\right)$. Acetylsalicylic acid was also found correlated with a worse odor identification $\left(r^{2}=0.09\right.$, 
A

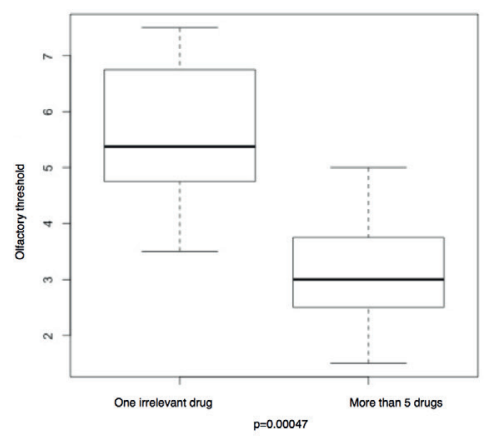

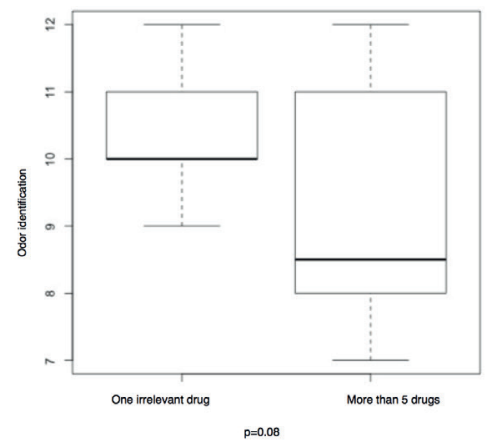

C

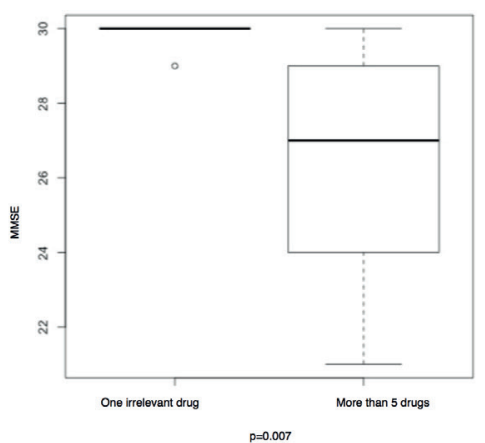

D

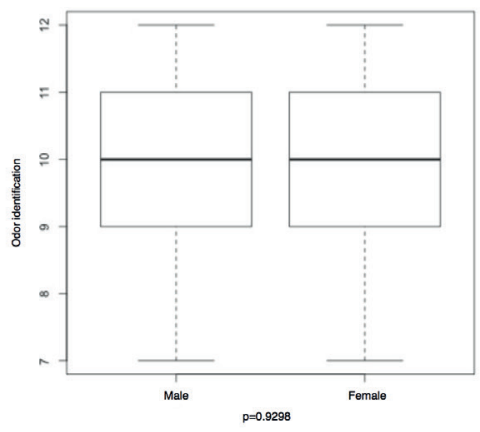

E

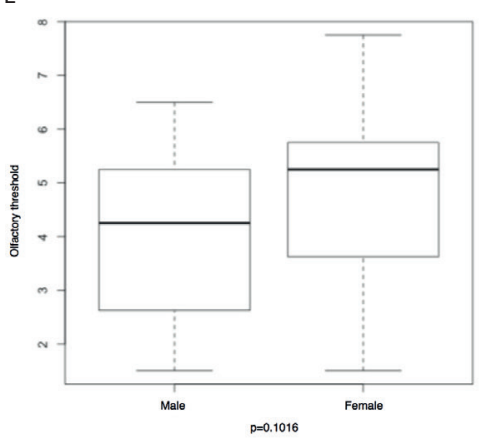

Figure 2. A: Boxplot of olfactory threshold by number of drugs taken. B: Boxplot of odor identification by number of drugs taken. C: Boxplot of MMSE by number of drugs taken. D: Boxplot of odor identification by sex. E: Boxplot of olfactory threshold by sex.

$\mathrm{p}=0.04)$, and so were potassium-sparing diuretics $\left(\mathrm{r}^{2}=0.12\right.$, $p=0.01$ ). Neither olfactory threshold nor odor identification correlated significantly with dicumarolics $(p=0.10$ and $p=0.85$, respectively), $a$-blockers $(p=0.12$ and $p=0.20)$, renin-angiotensin system inhibitors $(p=0.87$ and $p=0.23)$, sartans $(p=0.93$ and $p=0.85)$, benzodiazepines $(p=0.41$ and $p=0.61)$, statins $(p=0.09$ and $p=0.39$ ), proton pump inhibitors $(p=0.06$ and $p=0.86)$, or vitamin $D(p=0.37$ and $p=0.44)$. There were also no correlations between odor identification and calcium channel blockers ( $p=0.82$ ), ß-blockers ( $p=0.83$ ), or between olfactory threshold and potassium-sparing diuretics ( $p=0.05)$.

No differences were observed between males and females neither on odor identification nor on olfactory threshold (Figure 2d,e; Table 1).

The multivariate analysis conducted with a model involving all available variables to assess their influence on olfactory threshold showed that MMSE score, a-blockers, and acetylsalicylic acid significantly influenced participants' sense of smell in terms of their olfactory threshold (Table 3). In particular, the abovementioned drugs correlated with a worse olfactory threshold ( $p=0.04$ and $p=0.005$, respectively), while the MMSE score correlated with a better olfactory threshold $(p=0.04)$. When the multivariate analysis was conducted with a model involving all available variables to test their influence on odor identification, only acetylsalicylic acid revealed a significant inverse correlation $(p=0.006)$.

\section{Discussion}

Polypharmacotherapy can be associated with an increased risk of adverse effects ${ }^{(22)}$. Elderly people more frequently need several different types of medication because of their higher likelihood of suffering from more than one clinical disorder ${ }^{(10,23)}$. The reported prevalence of polypharmacotherapy in the elderly ranges from $13 \%$ to $92 \%{ }^{(22)}$. In particular, critically-ill elderly patients in hospitals and nursing homes are likely to take from 4 to 9 different drugs a day ${ }^{(10)}$. Among the adverse outcomes associated with polypharmacotherapy, olfactory dysfunction has also been reported ${ }^{(9,11)}$.

Probably the most interesting finding of the present study, conducted on a group of elderly volunteers with medium-tohigh MMSE scores and healthy sinonasal conditions, was that the number of different drugs they were taking correlated with a lower olfactory performance in the meaning of higher olfactory threshold. This result was confirmed when we compared the two sub-groups of volunteers with different number of medications taken. In fact, we found that the sub-group of subjects 
taking only 1 drug known to not influence olfaction showed a significantly better olfactory threshold than the other sub-group (taking more than 5 drugs) and a marginally better olfactory identification. Although the number of drugs taken correlated with a lower MMSE score, and this was also confirmed dividing the population in the two sub-groups based on the number of drugs consumed (Figure 1c), in our study population there was no correlation between MMSE score and olfactory performance (possibly owing to the small number of volunteers considered). There was, however, a significant correlation between MMSE score and better olfactory performance in the meaning of olfactory threshold on multivariate analysis, possibly justified by the fact that both parameters are usually age-related. In particular, with increasing age, the number of drugs taken usually rises and cognitive status usually declines. That only multivariate analysis revealed a correlation between MMSE score and olfactory threshold might be justified by the fact that, in order to be eligible for the present study, volunteers had to score more than 18 in the MMSE. The mean MMSE in our sample was actually $28 / 30$, indicating a very high cognitive status and relatively little variance ${ }^{(10)}$ across the group investigated.

Interestingly, unlike olfactory threshold, odor identification correlated with age, but not with drug consumption. This may be because olfactory thresholds exhibit a greater variance than odor identification scores, and the former reflect peripheral damage to the olfactory system more effectively than the latter. Finally, no associations came to light on univariate analysis between olfactory function and sex, weight, height or BMI. When these variables were included in a multivariate analysis, BMI showed to be marginally significant, though not quite at the $5 \%$ level, on affect olfactory threshold ( $p=0.057$ ) (Table 3 ). There is a paucity of literature on this matter and the picture is still unclear. A relatively recent study conducted on 24 young university staff members and students found that individuals' olfactory threshold for $\mathrm{n}$-butanol was better when fasting, and that participants with a lower BMI had a greater olfactory sensitivity than those with a higher one ${ }^{(24)}$. The authors concluded that their findings confirmed those of a study by Richardson and coworkers in obese subjects ${ }^{(25)}$ in which subjects with a BMI $>45$ had significantly lower odor identification scores than those with a BMI $<45$. The latter authors had suggested that either olfactory dysfunction had a role in the development of obesity, or morbid obesity was a factor in the decline in olfactory function in these individuals ${ }^{(25)}$. In this regard, recent studies found that about one in four morbidly obese patients were hyposmic and, after laparoscopic bariatric surgery, obese patients experienced a significant improvement in olfactory function ${ }^{(26,27)}$. There are still no clear explanations for the pathophysiological mechanis$\mathrm{ms}$ behind these findings ${ }^{(25,26)}$, although a relationship between olfactory modulation and nutritional status has been suggested in rodents ${ }^{(28)}$. In line with the above reports, BMI correlated with olfactory function (in terms of olfactory threshold) on multivariate analysis in our study too, although it was conducted not on obese people, but on a group of elderly individuals with a mean BMl of 25.5 (range 17.6-44.1).

At univariate analysis, both olfactory threshold and odor identification were influenced by some of the drugs taken by our sample population, while at multivariate analysis only olfactory threshold correlated significantly with some of the drugs involved. The types of medication that influenced olfaction were: potassium-sparing diuretics, antiplatelet drugs, a- and B-blockers, and calcium channel blockers. It may be that potassiumsparing diuretics interfere with olfactory receptor (OR) activity because ORs comprise a large class of G-protein-coupled receptors that, once activated, are capable of triggering neuronal activity ${ }^{(29)}$. As ORs are responsible for olfactory sensitivity, enabling us to discriminate between a variety of volatile and soluble molecules ${ }^{(29)}$, if potassium-sparing diuretics are able to influence ionic homeostasis at this level, they may also negatively influence OR activity, and the olfactory threshold as a result. In our group of elderly volunteers, antiplatelet drugs (acetylsalicylic acid) also negatively influenced both odor identification and olfactory threshold. Analyzing our study population in more detail revealed, however, that the subgroup of volunteers taking antiplatelet drugs was significantly older (mean age 79 as opposed to 72 for the subgroup not using this drug) $(p=0.004)$, and this age difference might reasonably explain this finding. Little is known about the effect of $a$ - and $ß$-blockers on olfaction. The presence of dopamine receptors in the olfactory neurons suggests that odor sensitivity may be modulated by neurotransmitters at this level. In fact, catecholamines are released into the mucus overlying the olfactory epithelium and have been found to modulate odor sensitivity via D2 dopamine receptors in rat ${ }^{(30)}$. A recent study by Lötsch and co-workers ${ }^{(9)}$ on a large sample identified an association between antagonistic targeting of the adrenoceptor a 1 A (ADRA1A) - such as a 1 adrenoceptor-blocking agents - and higher olfactory scores. It has been demonstrated that ADRA1A is the most common target among all those affected by the drugs reportedly influencing human olfaction, and it has been suggested that adrenergic activation enhances inhibitory transmission in the olfactory system. This is consistent with the anecdotal observation of a reversible disruption of the sense of smell after administration of the $a$-adrenoceptor agonist midodrine ${ }^{(30)}$. Given the effect of midodrine on human olfaction, a vasoconstrictive effect on the nasal mucosa, leading to dysosmia and then to a perceived dysgeusia, has also been hypothesized ${ }^{(31)}$. Olfaction is reportedly impaired by a-blockers in crickets. Pharmacological noradrenergic receptor blockade may interfere with aversive memory recall in olfactory conditioning ${ }^{(32)}$. It has also been demonstrated that modulation of the noradrenergic system can affect odor recognition by influencing odor memory in mice ${ }^{(33)}$. Finally, anosmia has been reported in humans taking 
B-blockers ${ }^{(34)}$. Though this is still only a speculation, it is reasonable to accept ADRA1A as a possible candidate drug target of relevance to human olfaction ${ }^{(31)}$. The present analysis also identified calcium channel blockers as being significantly correlated with a higher olfactory threshold (worse olfactory performance), and previous reports had described similar findings ${ }^{(35)}$. Although the present study indicated that some drugs affect olfactory sensitivity, we have to acknowledge that it concerned a relatively small number of volunteers, so no definitive conclusions can be drawn as yet. In particular, the effects of a-blockers on olfaction contrast with the findings of a previous study by Lötsch and coworkers on a large cohort of subjects ${ }^{(9)}$. This apparent discrepancy may be due both to the smaller sample considered in our study, and to the different approach used to assess the effects of these drugs on olfaction.

\section{Conclusion}

In conclusion, in the present study on a group of sinonasally healthy elderly volunteers, the number of drugs taken showed a significant correlation with both a worse olfactory performance (in terms of olfactory threshold) and worse MMSE scores. This investigation found that some drugs influence odor identification and/or olfactory threshold, but these results should be considered with caution, partly because elderly people's drug consumption is usually a medical necessity and unavoidable, and partly because more studies on larger samples of elderly volunteers are needed to explain some discordant results in the literature on this very intriguing and important topic as olfactory dysfunction has been demonstrated to induce a depressive and anxiolytic state, at least in mice ${ }^{(36)}$.

\section{Acknowledgement}

We thank Mrs Frances Coburn for the paper language-editing.

\section{Authorship contribution}

GO: conception, writing, discussion, supervision; ES: conception, data collection, writing; BS: statistics; $A B$ : data collection; PM: writing; GS: geriatric discussion; AM: supervision; EM: geriatric discussion, supervision; GM: conception, discussion, supervision.

\section{Conflict of interest}

No conflict of interest.

\section{References}

1. Doty RL, Shaman P, Applebaum SL, et al. Smell identification ability: Changes with age. Science 1984;226:1441-1443.

2. Mullol J, Alobid I, Mariño-Sánchez F, Quintó L, de Haro J, Bernal-Sprekelsen M, Valero A Picado C, Marin C. Furthering the understanding of olfaction, prevalence of loss of smell and risk factors: a population-based survey (OLFACAT study). BMJ Open 2012;2: e001256.

3. Ottaviano G, Lund VJ, Nardello E, et al. Peak nasal inspiratory flow: a useful and handy tool for the diagnosis of nasal obstruction in the elderly. Eur Arch Otorhinolaryngol 2014;271:2427-2431.

4. Brämerson $A$, Johansson $L$, Ek $L$, et al. Prevalence of olfactory dysfunction: the Skövde population-based study. Laryngoscope 2004;114:733-737.

5. Landis BN, Konnerth CG, Hummel T. A study on the frequency of olfactory dysfunction. Laryngoscope 2004;114:1764-1769.

6. Kern DW, Schumm LP, Wroblewski KE, et al. 2015. Olfactory thresholds of the U.S. Population of home-dwelling older adults: development and validation of a short, reliable measure. PLoS One 10:e0118589.

7. Mobley AS, Rodriguez-Gil DJ, Imamura F, Greer C. Aging in the olfactory system. Trends Neurosci 2014;37:77-84.

8. Ottaviano G, Frasson G, Nardello E, Martini A. Olfactory deterioration in cognitive disorders in the elderly. Aging Clin Exp Res 2016;28:37-45

9. Lötsch J, Daiker H, Hähner A, et al. Drugtarget based cross-sectional analysis of olfactory drug effects. Eur J Clin Pharmacol 2015;71:461-471.

10. Toffanello ED, Inelmen EM, Imoscopi A et al. Taste loss in hospitalized multimorbid elderly subjects. Clin Interv Aging 2013:8:167-174.

11. Doty RL, Bromley SM. Effects of drugs on olfaction and taste. Otolaryngol Clin North Am 2004:37:1229-1254

12. Hopkins C, Gillett S, Slack R, et al. Psychometric validity of the 22-item Sinonasal Outcome Test. Clin Otolaryngol 2009:34:447-454.

13. Magni E, Binetti G, Bianchetti R, et al. MiniMental State Examination: a normative study in the Italian elderly population. Eur J Neurol 1996:3:198-202.

14. Sami AS, Scadding GK, Howarth P. A UK community-based survey on the prevalence of rhinosinusitis. Clin Otolaryngol. 2018:43:76-89.

15. Ottaviano G, Scadding GK, lacono V, Scarpa B, Martini A, Lund VJ. Peak nasal inspiratory flow and peak expiratory flow. Upright and sitting values in an adult population. Rhinology 2016;54:160-163.

16. Schöpf V, Kollndorfer K, Pollak M, Mueller CA, Freiherr J. Intranasal insulin influences the olfactory performance of patients with smell loss, dependent on the body mass index: A pilot study. Rhinology 2015;53:371378.

17. Hummel T, Konnerth CG, Rosenheim K, Kobal G. Screening of olfactory function with a four-minute odor identification test: reliability, normative data, and investigations in patients with olfactory loss. Ann
Otol Rhinol Laryngol 2001.110:976-981.

18. Hummel T, Whitcroft $K L$, Andrews $P$, et al. Position paper on olfactory dysfunction. Rhinol Suppl. 2017;54:1-30.

19. Ottaviano G, Cantone E, D'Errico A, et al. Sniffin' Sticks and olfactory system imaging in patients with Kallmann syndrome. Int Forum Allergy Rhinol 2015;5:855-861.

20. Ottaviano G, Staffieri A, Stritoni $P$, et al. Nasal dysfunction induced by chlorinate water in competitive swimmers. Rhinology 2012;50:294-298.

21. Miller A. Subset selection in regression. Chapman \& Hall/CRC 2002.

22. Maggiore RJ, Gross CP, Hurria A. Polypharmacy in older adults with cancer. Oncologist 2010;15:507-522.

23. Jorgensen $T$, Johansson $S$, Kennerfalk $A$, et al. Prescription drug use, diagnoses, and healthcare utilization among the elderly. Ann Pharmacother 2001;35:1004-1009.

24. Stafford LD, Welbeck K. High hunger state increases olfactory sensitivity to neutral but not food odors. Chem Senses 2011;36:189198.

25. Richardson BE, Vander Woude EA, Sudan $\mathrm{R}$, et al. Altered olfactory acuity in the morbidly obese. Obes Surg 2004;14:967-969.

26. Holinski F, Menenakos C, Haber G, et al. Olfactory and gustatory function after bariatric surgery. Obes Surg 2015;25:2314-2320.

27. Hanci D, Altun $H$, Altun $H$, et al. Laparoscopic sleeve gastrectomy improves olfaction sensitivity in morbidly obese patients. Obes Surg2016;26:558-562.

28. Thiebaud N, Johnson MC, Butler JL, et al. Hyperlipidemic diet causes loss of olfactory 
sensory neurons, reduces olfactory discrimination, and disrupts odor-reversal learning. J Neurosci 2014;34:6970-6984.

29. Ottaviano G, Zuccarello D, Frasson G, et al. Olfactory sensitivity and sexual desire in young adult and elderly men: an introductory investigation. Am J Rhinol Allergy 2013,27:157-161.

30. Vargas G, Lucero MT. Dopamine modulates inwardly rectifying hyperpolarization-activated current (Ih) in cultured rat olfactory receptor neurons. J Neurophysiol 1999:81:149-158.

31. Young, T.M. and Mathias, C.J. Taste and smell disturbance with the alpha-adrenoceptor agonist midodrine. Ann. Pharmacother 2004:38:1868-1870.

32. Mizunami M, Unoki S, Mori Y, et al. Roles of octopaminergic and dopaminergic neurons in appetitive and aversive memory recall in an insect. BMC Biol 2009;7:46.

33. Veyrac A, Nguyen $V$, Marien $M$, et al. Noradrenergic control of odor recognition in a nonassociative olfactory learning task in the mouse. Learn Mem 2007;14:847-854.

34. Vital Durand M. Recurrent anosmia under beta-blockers. Presse Med 1985;14:2064.

35. Ackerman BH, Kasbekar N. Disturbances of taste and smell induced by drugs. Pharmacotherapy 1997;17:482-496.

36. Ahn S, Shin HW, Mahmood U, Khalmuratova R, Jeon SY, Jin HR, Choi JS, Kim HS, Kim DW Chronic anosmia induces depressive behavior and reduced anxiety via dysregulation of glucocorticoid receptor and corticotropin-releasing hormone in a mouse model. Rhinology 2016;54:80-87.

37. Eibenstein A, Fioretti AB, Lena C, Rosati N Ottaviano I, Fusetti M. Olfactory screening test: experience in 102 Italian subjects. Acta Otorhinolaryngol Ital 2005;25:18-22.

38. Hummel T, Kobal G, Gudziol H, Mackay-Sim A. Normative data for the "Sniffin' Sticks" including tests of odor identification, odor discrimination, and olfactory thresholds: an upgrade based on a group of more than
3,000 subjects. Eur Arch Otorhinolaryngol 2007:264:237-243.

Giancarlo Ottaviano, MD, PhD

Dept. of Neurosciences DNS

Otolaryngology Section

University of Padova

Via Giustiniani 2

35128 Padova

Italy

Tel. +390498212029

$\mathrm{Fax}+390498213113$

E-mail: giancarlo.ottaviano@unipd.it 